\title{
Norois
}

Environnement, aménagement, société

$194 \mid 2005 / 1$

Les milieux polaires et subpolaires de l'Atlantique

\section{Pour une approche paysagère des espaces polaires}

L'exemple du Spitsberg occidental

For a landscape approach of the polar regions, the example of West Spitsbergen

Madeleine Griselin et Serge Ormaux

\section{(2) OpenEdition}

Journals

Édition électronique

URL : http://journals.openedition.org/norois/682

DOI : $10.4000 /$ norois. 682

ISBN : 978-2-7535-1542-0

ISSN : $1760-8546$

Éditeur

Presses universitaires de Rennes

Édition imprimée

Date de publication : 1 mars 2005

Pagination : 109-123

ISBN : 978-2-7535-0100-3

ISSN : 0029-182X

Référence électronique

Madeleine Griselin et Serge Ormaux, «Pour une approche paysagère des espaces polaires », Norois [En ligne], 194 | 2005/1, mis en ligne le 13 août 2008, consulté le 30 avril 2019. URL : http:// journals.openedition.org/norois/682; DOI : 10.4000/norois.682

Ce document a été généré automatiquement le 30 avril 2019.

(c) Tous droits réservés 


\title{
Pour une approche paysagère des espaces polaires
}

\author{
L'exemple du Spitsberg occidental \\ For a landscape approach of the polar regions, the example of West Spitsbergen
}

\author{
Madeleine Griselin et Serge Ormaux
}

\section{NOTE DE L'ÉDITEUR}

Cet article a été reçu le 13 décembre 2004 et définitivement accepté le 23 février 2005.

1 Les rapports entre paysage et géographie sont depuis longtemps marqués par une étrange dialectique d'engouement et de méfiance. Dans La Face de la terre, Ph. et G. Pinchemel (1997) montraient comment la célébration d'une géographie-science des paysages conduisit à un procès en tromperie, puis à un divorce, avant de donner lieu, au moins pour certains, à une réhabilitation sur la base de valeurs nouvelles. Dans le premier volume de la Géographie universelle, Roger Brunet illustrait cette relation ambiguë en affirmant que « les paysages n'ont pas de sens » mais en concédant que «si le paysage ne fait pas signe ", au moins, "il indique », et que s'il faut le «contester [...] il révèle à qui sait le regarder »! (Brunet et Dollfus, 1990). Dans la littérature scientifique sur les espaces polaires, le paysage a souvent été considéré avant tout comme un support d'illustration, dont l'efficacité était amplifiée par l'impression de grande lisibilité offerte par les topographies dénudées et par la forte traduction visuelle des processus analysés. Cette forte prégnance paysagère du fait polaire et la force émotionnelle de la plupart des clichés utilisés ont pu faire oublier qu'entre les processus dont ils témoignent et l'émotion esthétique qu'ils suscitent, ces paysages pouvaient aussi être des objets de connaissance.

2 Mais la recherche d'aujourd'hui n'est pas exempte d'équivoque et de glissements. Si le lien établi entre géosystème paysage a été productif, il a conduit également à une insuffisante distinction entre les systèmes fonctionnels produisant le paysage et les 
structures physionomiques du paysage visible. La tentation d'assimiler la science du paysage à l'écologie du paysage a été renforcée encore par le développement des outils de télédétection. En effet, la vue du dessus parle peu et mal du paysage tel que le voient les habitants ou les visiteurs d'un lieu ; par facilité ce paysage fut souvent laissé en apanage aux approches culturalistes. Certes, le paysage n'est pas signe, et encore moins langage, mais il est information et c'est dans ce flux d'information émis par de multiples forces et processus en interaction que réside un gisement, exploitable à de nombreux niveaux.

\section{Nouvelle donne technique et nouvelle demande sociale vis-à-vis du paysage en général et des paysages polaires en particulier}

3 Le principe d'une mobilisation systématique de l'information paysagère a été développé dès les années 1970 par plusieurs écoles paysagères et a permis de dégager des principes encore incontournables tels que ceux de l'observation tangentielle, du continuum spatial, de l'approche échantillonnée (Ormaux, 1987). Mais la difficulté d'exploiter de gros corpus de clichés et de fiches d'analyses a bien souvent compromis l'opérationnalité de la démarche. Aujourd'hui, les possibilités de gestion offertes par les logiciels de banques d'images, la maîtrise du géoréférencement facilitée par les SIG, et les diverses possibilités d'expression de la réalité paysagère permises par les outils multimédia ont changé la donne (Griselin et Ormaux, 2000, 2001b).

4 Par ailleurs, la demande sociale en matière de paysage a considérablement évolué. D'une part, les préoccupations écologiques ont glissé de l'univers strictement environnementaliste vers celui du paysage considéré comme un objet en soi. D’autre part, la sensibilité patrimoniale s'est dégagée du seul domaine des œuvres d'art ou des objets manufacturés pour intégrer les paysage eux-mêmes, considérés comme œuvre des hommes. L'information paysagère, principalement sous forme de collections d'images devient dans ce contexte une information partagée, un support de médiation autour duquel décideurs, experts et habitants pourront échanger leurs points de vue et envisager des hypothèses.

5 Contrairement à ce que l'on pourrait croire, les contrées polaires n'échappent pas à cette évolution. Après avoir été la chasse gardée des découvreurs, puis celle des chercheurs, les pôles sont maintenant considérés par nos contemporains comme un patrimoine planétaire dont le devenir est l'affaire de tous. Le regain d'intérêt dont ils sont l'objet accroît les pressions de toute sorte et multiplie les représentations. Vus par certains comme traceurs particulièrement sensibles du changement climatique, convoités par d'autres comme gisements souterrains ou routes maritimes libérés par le réchauffement, regardés enfin comme espaces de rêve désormais atteignables par les nouveaux touristes de l'extrême, ils méritent plus que jamais d'être appréhendés dans la spécificité de leurs paysages multiples.

6 Entre la vision réductrice du "grand blanc» forgée par le mythe, la connivence chamanique des peuples du froid avec leur cadre de vie et le calcul intéressé des opérateurs économiques, il y a place, pensons-nous, pour une émergence scientifique des paysages polaires. 


\section{Une géographie des paysages en milieu polaire}

7 Une carte d'ensemble de la toundra arctique a été récemment réalisée et a montré la grande diversité de cette formation végétale cernant l'océan Glacial Arctique (Raynolds et Walcker, 2004); de nombreux travaux géomorphologiques, menés ici ou là, laissent entrevoir la multiplicité des combinatoires dont ces contrées sont le siège (André, 1993). L'approche paysagère demeure quant à elle peu pratiquée alors que le monde polaire présente tout à la fois une grande ampleur de la visibilité (du fait de la faible présence de la végétation), une large palette de compositions paysagères, et une très grande variabilité de ces dernières aux échelles de la journée, de l'année et du siècle, que ce soit sur la terre ou sur la banquise. Ces dispositions particulières donnent plus qu'ailleurs la possibilité d'étudier les paysages dans le cadre d'une géographie régionale des configurations paysagères. Cette offre de paysages, appréhendée à travers le concept de « paysage visible » - au sens de potentiellement offert à la vue - est une des dimensions de la connaissance de ces espaces.

8 Le caractère épuré et minéral des espaces polaires facilite la mise en œuvre d'analyses critériées des paysages et l'établissement de typologies. Le cédérom que nous présentons ici ouvre le champ de ce type d'approche et se veut une pédagogie de l'analyse des paysages polaires, à travers les pratiques de l'échantillonnage systématique.

9 Le principe de l'hypertexte autorise un discours multi-dimensionnel particulièrement adapté à l'approche paysagère. Comment en effet parler de paysage en se limitant à la seule matérialité, au seul présent et à la seule vision immobile? A partir des paysages potentiellement visible dans la baie du Roi, nous proposons justement de croiser et d'articuler les regards (fig. 1). 
Figure 1 : Les trois dimensions de l'approche paysagère et les quatre domaines informationnels abordés par le cédérom

The three dimensions of the landscape approach and the four informational domains taken up by the $C D$ Rom

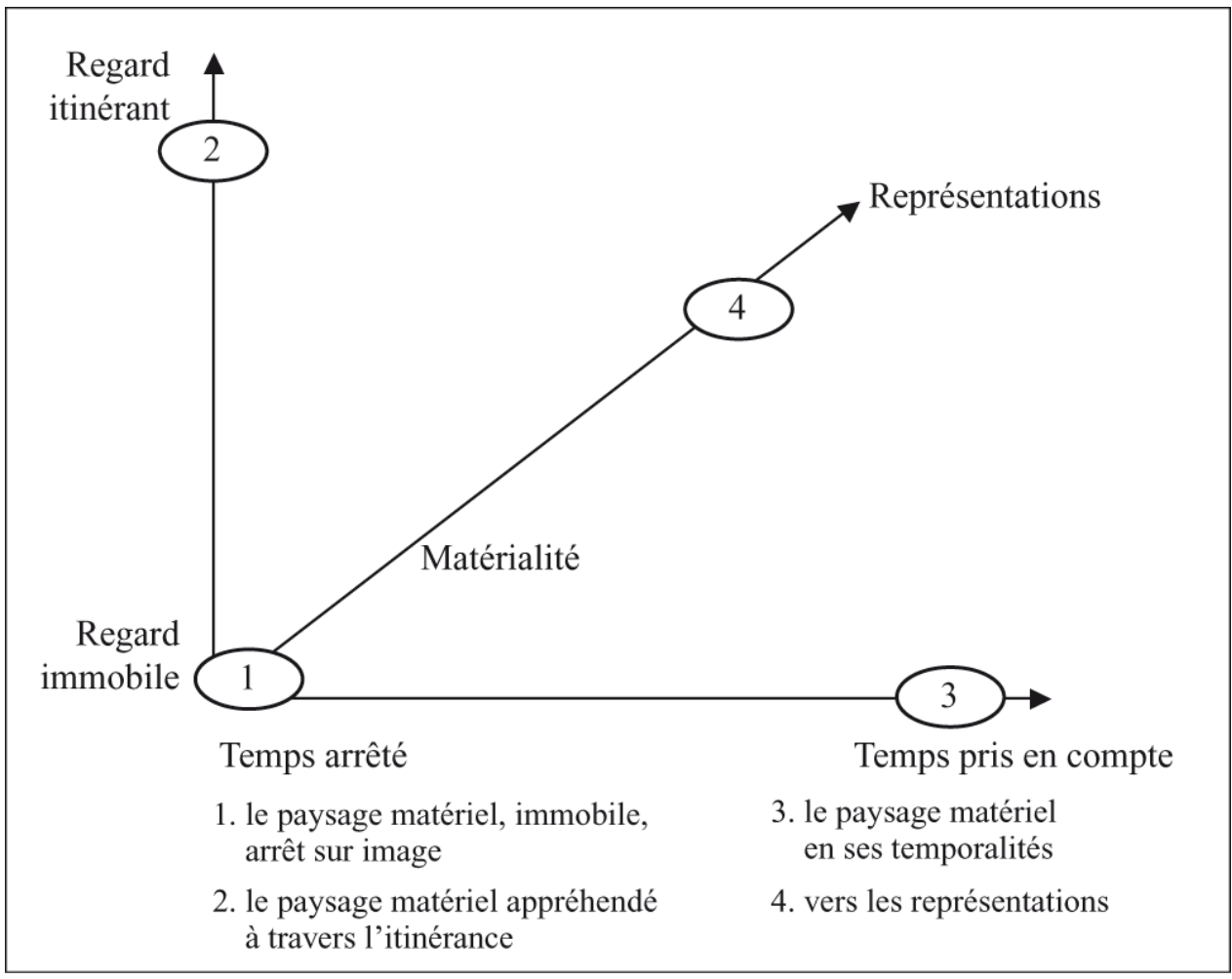

On part d'une analyse physionomique, strictement objectale, réalisée à un instant $t$ grâce à une procédure d'échantillonnage photographique. La focalisation sur le bassin du glacier Lovén Est (Spitsberg, $79^{\circ} \mathrm{N}$ ) permet de prendre en compte les trois géosystèmes fondamentaux de la côte ouest, le sandur, la moraine et le glacier lui-même, de les combiner entre eux, et de les associer dans les scènes paysagères à des perspectives ouvertes vers le fjord et vers l'encadrement montagneux.

La banque d'images obtenue appréhende ainsi le continuum du paysage à travers le double principe de la vision depuis le sol, fondamentalement intégrative, et de l'échantillonnage spatial, qui garantit tout à la fois la représentativité du corpus et son exploitation géoréférencée. Ici apparaît la diversité et l'organisation spatiale des paysages du Lovén Est et de la baie du Roi, exprimée à l'aide de modèles graphiques (Brossard et al., 1998).

12 Mais le paysage ne saurait être réduit à des regards immobiles, fussent-ils pluridirectionnels et fussent-ils réitérés. Si l'approche scientifique du paysage visible ne peut se satisfaire de quelques points de vue panoramiques judicieusement choisis, elle ne peut non plus tout à fait se réduire à la juxtaposition de l'information dans un document synoptique. Précisément parce que la vision paysagère au sol n'est pas synoptique et qu'elle se déploie le plus souvent dans la linéarité (éventuellement zigzagante) du déplacement. C'est ce dont cherche à rendre compte le module consacré aux cheminements, ici pédestres, et qui relie les paysages dans des séquences plus ou moins complexes le long de transects-types ou de déambulations plus spontanées. 
13 Même si l'on reste ici encore sur le versant objectal du paysage, on se rapproche de la pratique de l'espace-paysage, à travers le déplacement d'un chercheur, d'un randonneur, ou d'un des rares habitants de la contrée. La technique utilisée permet de simuler des déplacements à partir d'une interface graphique et de voir s'enchaîner sur l'écran les séquences paysagères. Ces dernières apparaissent particulièrement heurtées, en termes de combinaisons d'objets et en termes d'échelles spatiales.

Restituer aux paysages leur dynamique, c'est également prendre en compte leur dimension temporelle. Plus que d'autres, les paysages des contrées polaires affichent une forte variabilité temporelle. Les évolutions à long terme peuvent être prises en compte par la comparaison de corpus-échantillons réalisés à une trentaine d'années d'écart, en liaison avec des suivis de documents projectionnels. Mais les changements rapides et les cycles saisonniers peuvent faire l'objet de dispositifs de veille photographique selon des principes d'échantillonnage temporel. Le module «Au fil du temps » ouvre des pistes dans ce sens et montre le permanent renouvellement des paysages polaires, à la fois par la modification de leurs objets constitutifs (neige, cours d'eau, glace, toundra, etc.) et par la modification des conditions de leur vision (nébulosité, position du soleil).

Enfin, d'autres liens permettent de relier toutes ces approches physionomiques et matérielles avec le domaine des représentations: images anciennes construisant les mythes, discours touristique d'aujourd'hui, regards d'artistes. Cela fait aussi partie de la réalité paysagère, puisque cela boucle sur les processus physiques à travers les décisions et les actions humaines.

\section{Un cédérom pour une navigation spatio-temporelle dans le paysage arctique}

Prix « Enseignement de la recherche » au Festival du film de chercheur de Nancy en 2002, le cédérom trilingue (français, anglais, espagnol) «Paysages vus du sol: principes d'analyse paysagère, application en milieu arctique (Spitsberg) » (Griselin et Ormaux, 2005), présente, à partir d'une étude menée au Spitsberg $\left(79^{\circ} \mathrm{N}\right)$, les principes d'analyse des paysages pratiquée par les géographes bisontins à partir de corpus de photographies au sol (Griselin et Ormaux, 2001a).

La banque d'images géographiquement référencées réalisée au cours du programme d'étude est accessible grâce à une interface cartographique interactive. Ce cédérom n'est donc pas seulement un "discours de la méthode ", il permet de rendre au paysage de la Baie du Roi sa continuité spatiale et temporelle.

\section{Une architecture en trois niveaux}

Sur fond d'ambiance polaire hivernale, avec une signalétique inspirée des inuksuit ou "montreurs de chemins ", empilement de pierres édifiés par les Inuit, servant de repères dans l'espace et balisant les paysages de l'Arctique, le cédérom s'ouvre sur une spirale de pierres, invitant à une découverte, linéaire ou non, des cinq grands modules développés (fig. 2). 
Figure 2 : L'écran de parcours du cédérom The CD-Rom navigation interface

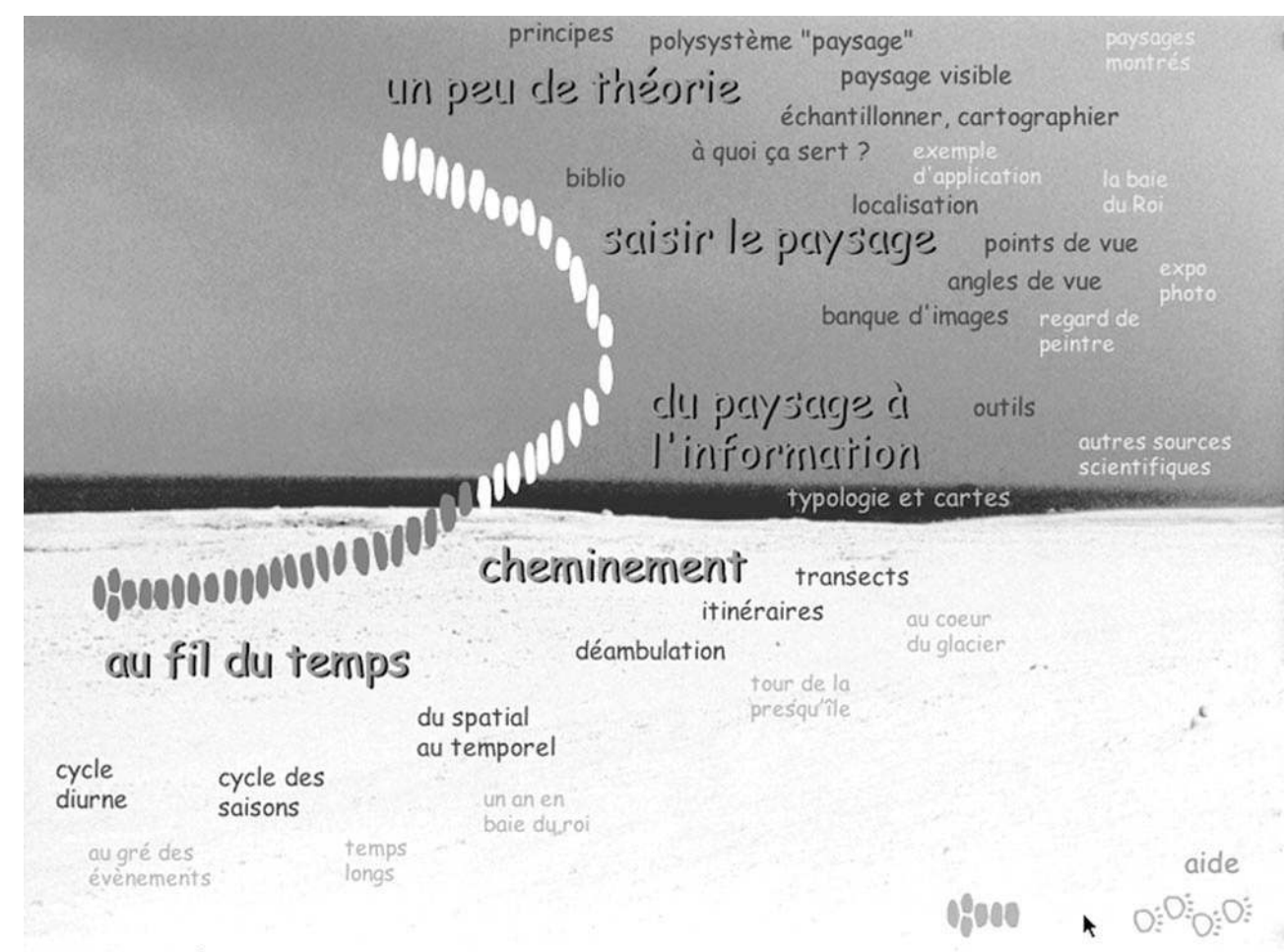

L'architecture repose sur trois niveaux : 5 grands thèmes sont déclinés en 18 modules et constituent le corps du cédérom, tandis qu'en troisième niveau, onze évocations complètent l'information de base par des exemples, des généralisations, des changements d'échelle, des pistes de travail, des regards différents, etc.

«Un peu de théorie » expose en cinq modules les fondements de l'analyse paysagère. Un sixième point est constitué par une bibliographie abondante et exportable de références concernant le paysage et l'Arctique.

21 «Saisir le paysage » situe l'étude; il rend compte des modes d'échantillonnage spatial et angulaire et permet l'accès à la banque d'images.

«Du paysage à l'information" aborde les outils et le traitement de l'information paysagère et, à travers quelques exemples, propose une typologie et une cartographie des paysages rencontrés.

Les deux derniers thèmes "Cheminement» et "Au fil du temps" permettent la navigation dans la banque d'image spatio-temporelle.

\section{Un environnement très particulier}

24 L'expérience présentée nous conduit dans l'univers polaire de l'archipel du Svalbard. Ici, le système producteur du paysage se réduit, pour l'essentiel, à la composante abiotique. Situé sur la côte nord-ouest du Spitsberg occidental, le bassin du glacier Lovén Est s'étend sur la presqu'île de Brøgger, en rive sud de la baie du Roi (Kongsfjord) par $79^{\circ}$ Nord, à six kilomètres de la petite station norvégienne de Ny Ålesund. Le Lovén Est est par ailleurs connu et étudié en profondeur grâce à la proximité d'une base de recherche française, fréquentée depuis quarante ans. 

englacé à $60 \%$. Comme la plupart des glaciers du Spitsberg, le Lovén Est est en pleine phase de retrait et recule derrière un appareil morainique, aujourd'hui surdimensionné, correspondant au maximum du Petit Âge Glaciaire (Griselin et al., 1995). Ce recul est considérable (800 m en moins de 100 ans dont $400 \mathrm{~m}$ entre 1970 et 1990). Le vallum morainique bute contre une barre calcaire, véritable barrière aux écoulements, dans laquelle les torrents issus du glacier ont forcé deux passages en canyon: c'est là toute l'originalité hydrologique mais aussi paysagère de ce bassin par rapport à ses voisins (fig. 3).

Figure 3 : Le bassin du Lovén Est (photo aérienne $\odot N P I)$ The East Lovén basin (aerial photo @NPI)

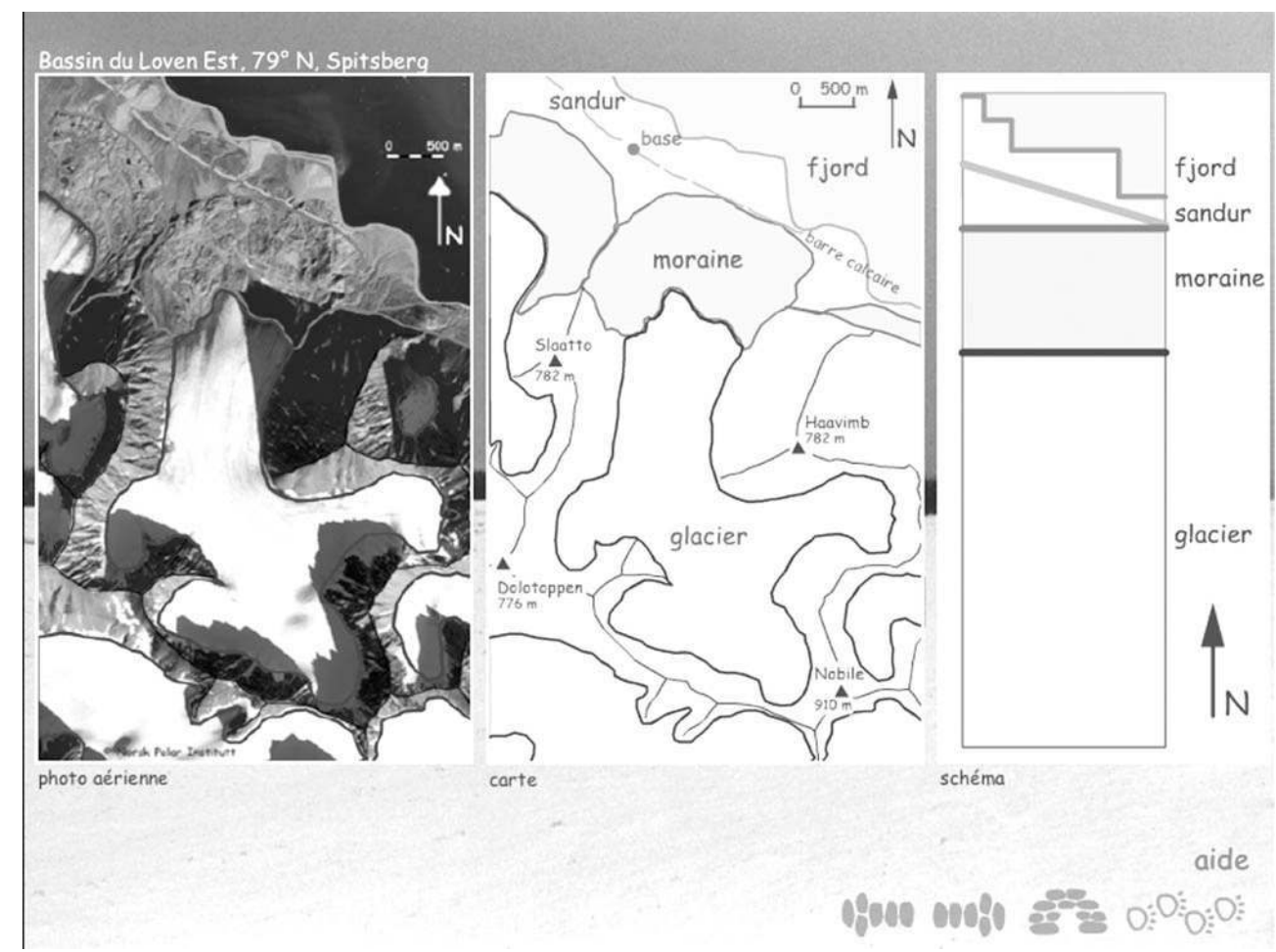

La presqu'île de Brøgger a une architecture caractéristique commune aux îles et péninsules de la côte nord-ouest du Spitsberg. Un axe montagneux central dominant un piémont sédimentaire en gradins. Les glaciers ont utilisé préférentiellement les lignes de faiblesse naturelle de la presqu'île, d'orientation NNE-SSO: ils renforcent l'allure compartimentée du relief de la presqu'île en isolant de part et d'autre de l'arête axiale des bassins individuels.

À la fois semblable à ses voisins et pourtant si différent, le bassin du Lovén Est se compose de quatre grandes unités paysagères :

- une ligne de crêtes lui conférant son allure d'amphithéâtre : elle culmine au pic Nobile au sud, tandis que, tels deux bastions, les pics Håvimb, à l'est, et Slåtto, à l'ouest, la limitent vers le nord;

- le glacier lui-même : s'étirant sur 7 kilomètres, il est constitué de trois affluents de glace issus des cirques convergeant vers $350 \mathrm{~m}$ d'altitude pour former la langue terminale, large d'un kilomètre, coulant du sud au nord, et butant sur un affleurement calcaire apparent à l'ouest, ce qui lui donne une dissymétrie certaine au front ; 
- la zone morainique - milieu pionnier par excellence, puisque récemment déglacé - structurée par les affleurements calcaires et cernée par un puissant vallum ;

- le sandur, entre moraine et fjord : étendue plane constituée de matériaux détritiques façonnés par les dynamiques fluvio-glaciaire et marine, il est traversé par une barre calcaire (NO-SE) contre laquelle vient buter le vallum.

28 Au-delà du bassin au sens strict, le fjord du Roi (Kongsfjord), large de six à huit kilomètres a été creusé par le glacier à front marin qui le ferme au sud-est. Sur l'autre rive du fjord s'étendent des unités paysagères structurellement proches de celles de la presqu'île de Brøgger où alternent des montagnes aux formes pyramidales caractéristiques de l'érosion polaire, berceaux de petits glaciers allant parfois jusqu'à la mer, ou nunataks émergeant de la calotte, vestige d'un inlandsis puissant qui aurait couvert l'intégralité de l'archipel à la période glaciaire. Limité à l'ouest par le cap Mitra, finistère de cette région ouverte sur la mer du Groenland, ces paysages «d'outre fjord» répondent parfaitement (comme la presqu'île de Brøgger du reste) à l'appellation donnée par Ch.-P. Péguy (1969) de "montagnes qui flottent sur la mer ", évoquant l'allure tout à la fois marine et hautealpine de reliefs culminant à peine à $1000 \mathrm{~m}$ d'altitude.

À ce décor physique, il faut ajouter quelques caractéristiques climatiques influant sur le paysage. Le climat polaire océanique confère à la côte nord-ouest du Spitsberg, en toutes saisons, une extrême variabilité journalière du temps météorologique et donc des ambiances climatiques qui contribuent à la variation temporelle du paysage (Joly, 2004). De plus, la neige (de septembre à juillet), puis la prise en glace du fjord (de décembre à juin), sont des éléments climatiques apportant une grande variation saisonnière dans les paysages de cette région.

Enfin, constante de l'Arctique, le balancement des saisons qui plonge le Spitsberg dans la nuit polaire de novembre à février et dans le jour permanent d'avril à fin août, est une donnée importante de la variation temporelle de la lumière et donc des paysages (Griselin et Ormaux, 2003).

\section{Une banque d'images reposant sur un échantillonnage systématique}

31 Les points de prise de vue ont été déterminés de manière systématique, la configuration du bassin du Lovén Est a guidé l'établissement du plan d'échantillonnage selon une logique de transects : 103 points au total ont été positionnés sur les sept transects. La densité des points varie en fonction des caractéristiques de chaque zone: la distance nord-sud entre deux points est de $250 \mathrm{~m}$ sur le sandur, $125 \mathrm{~m}$ dans la moraine et au front du glacier, $250 \mathrm{~m}$ sur le glacier, le long de trois transects seulement, $500 \mathrm{~m}$ dans le haut du glacier (fig. 4). 
Figure 4 : L'échantillonnage des points de prise de vue The Sampling of the shot's locations

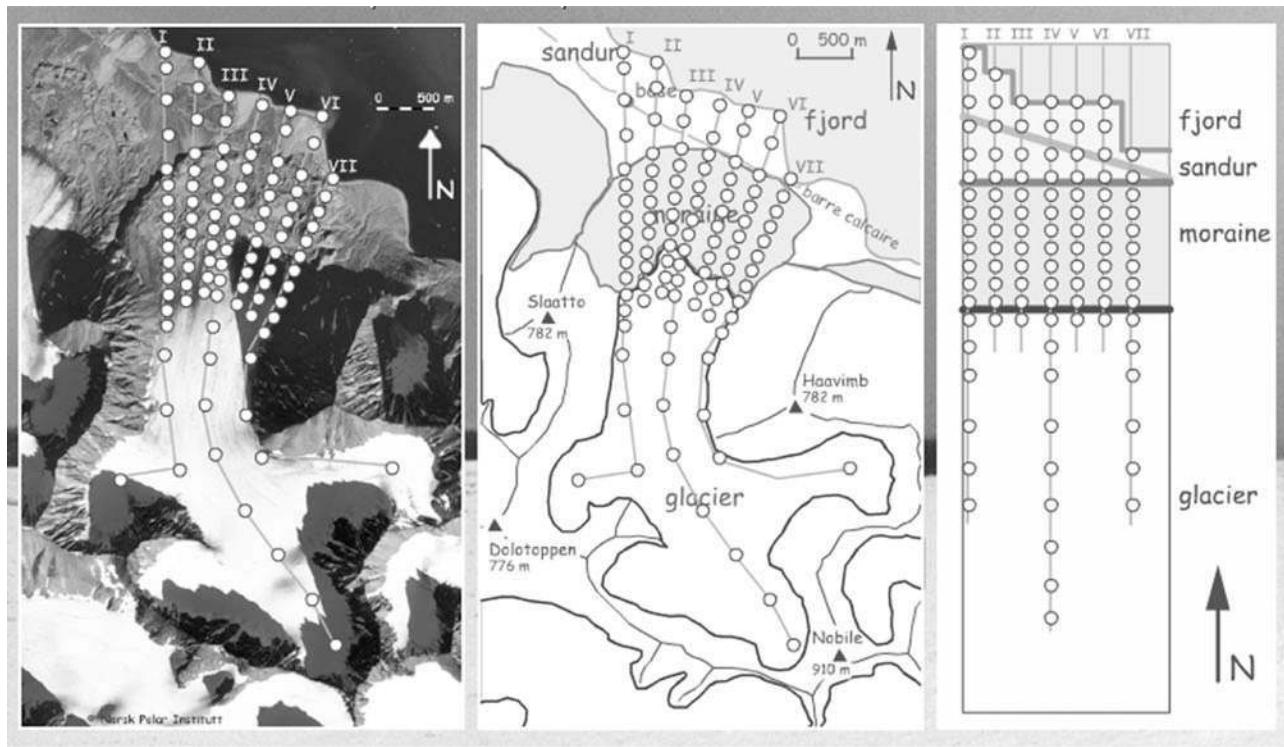

En chaque point, un principe d'échantillonnage angulaire est appliqué : trois prises de vue de $45^{\circ}$ rendent compte des $360^{\circ}$ de la scène paysagère. Les clichés sont pris à l'horizontale (fig. 5). En chaque point, un tirage aléatoire préalable détermine la direction de la première prise de vue entre 0 et $120^{\circ} \mathrm{du}$ nord. La deuxième direction de prise de vue est à $120^{\circ}$ de la première, la troisième à $240^{\circ}$. Le tirage au sort est répété pour chaque point du bassin. Un quatrième cliché est réalisé verticalement en direction du sol. Les quatre clichés de chaque point constituent une banque de 400 images qu'on peut atteindre grâce à une interface cartographique. 
Figure 5 : L'échantillonnage des angles de prise de vue The sampling of the shot's angles

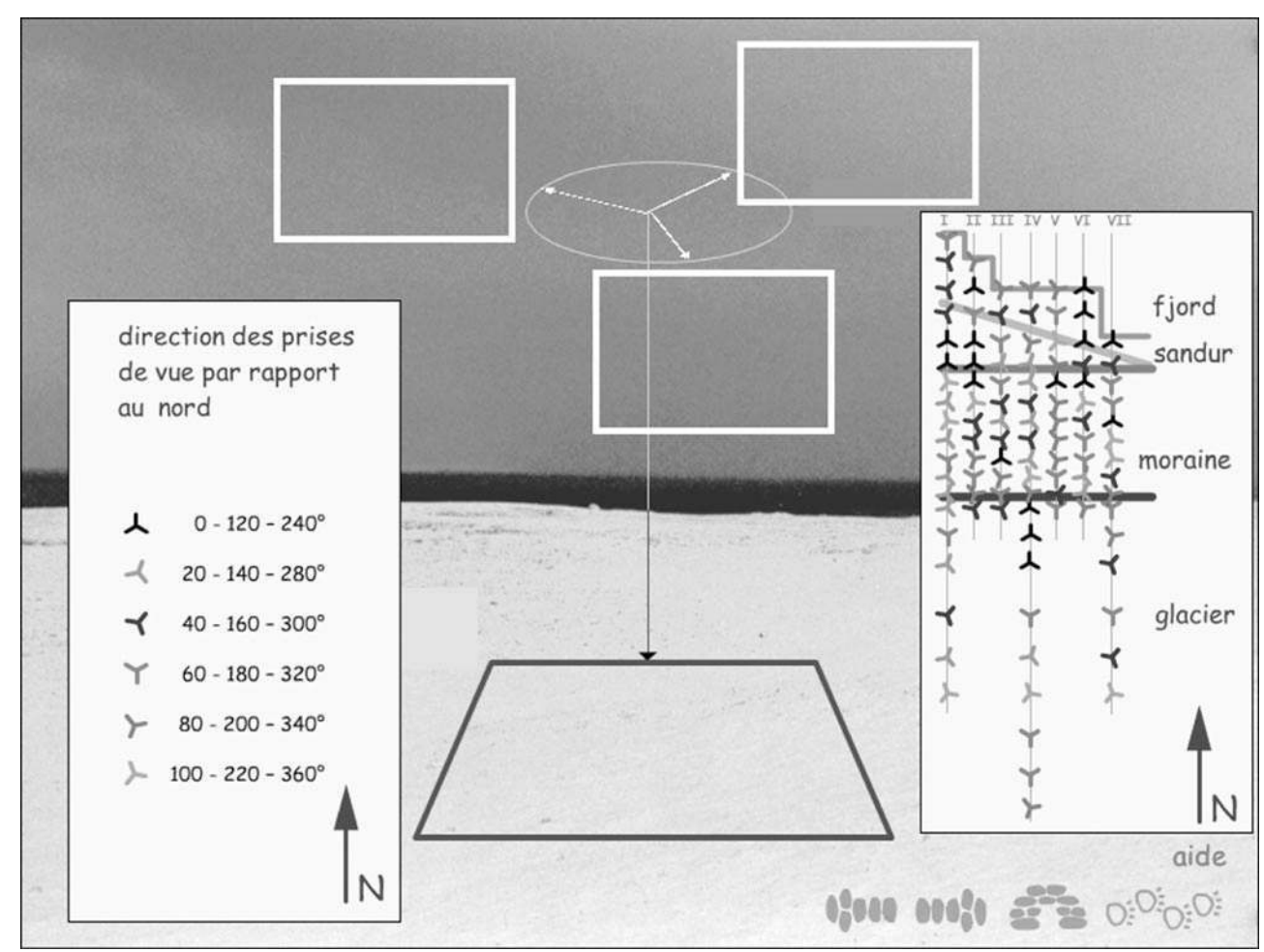

\section{Du paysage à l'information}

Un outil informatique de saisie et de traitement facilite l'analyse des photographies de paysage et permet de renseigner les clichés en ce qui concerne la composition, la colorimétrie, les éléments d'image, les objets du paysage (Borodkine et al., 2001). La lumière et la couleur sont appréhendées à travers les trois paramètres fondamentaux que sont la tonalité (couleur proprement dite), la saturation (intensité de la couleur), la luminance (intensité lumineuse). Ces paramètres sont mesurés sur l'image dans son ensemble mais peuvent l'être également sur des objets ou groupes d'objets du paysage. Appliquées à des clichés d'une même scène paysagère, pris à différents moments (saisons, jours, heures), ces mesures automatiques de la lumière et de la couleur permettent des comparaisons objectives pour l'étude de la variation temporelle des paysages.

Le traitement des images paysagères permet d'établir des typologies du paysage visible depuis chaque point de l'espace étudié. Les six éléments de base du paysage de la baie du Roi sont inventoriés sur chaque cliché (outre-fjord, fjord, sandur, montagne, glacier, moraine) et totalisés par secteur directionnel ainsi que pour l'ensemble des trois vues.

Par ailleurs, chaque paysage est une composition complexe qui varie selon le point et la direction d'observation. Dans notre exemple, les six éléments de base pris en compte pour le bassin du Lovén Est offrent 41 combinaisons possibles. Dix combinaisons sont particulièrement représentées au sein des clichés et constituent la matière de la typologie. 
36 En considérant les combinaisons paysagères de chaque cliché, direction par direction, on obtient une carte des combinaisons visibles en chaque point de prise de vue, dans les trois secteurs directionnels (fig. 6).

Figure 6 : Typologie et cartographie des paysages vus depuis les 103 points d'échantillonnage du Lovén Est

Typology and mapping of the landscapes seen from the 103 points sampled on the East Lovén

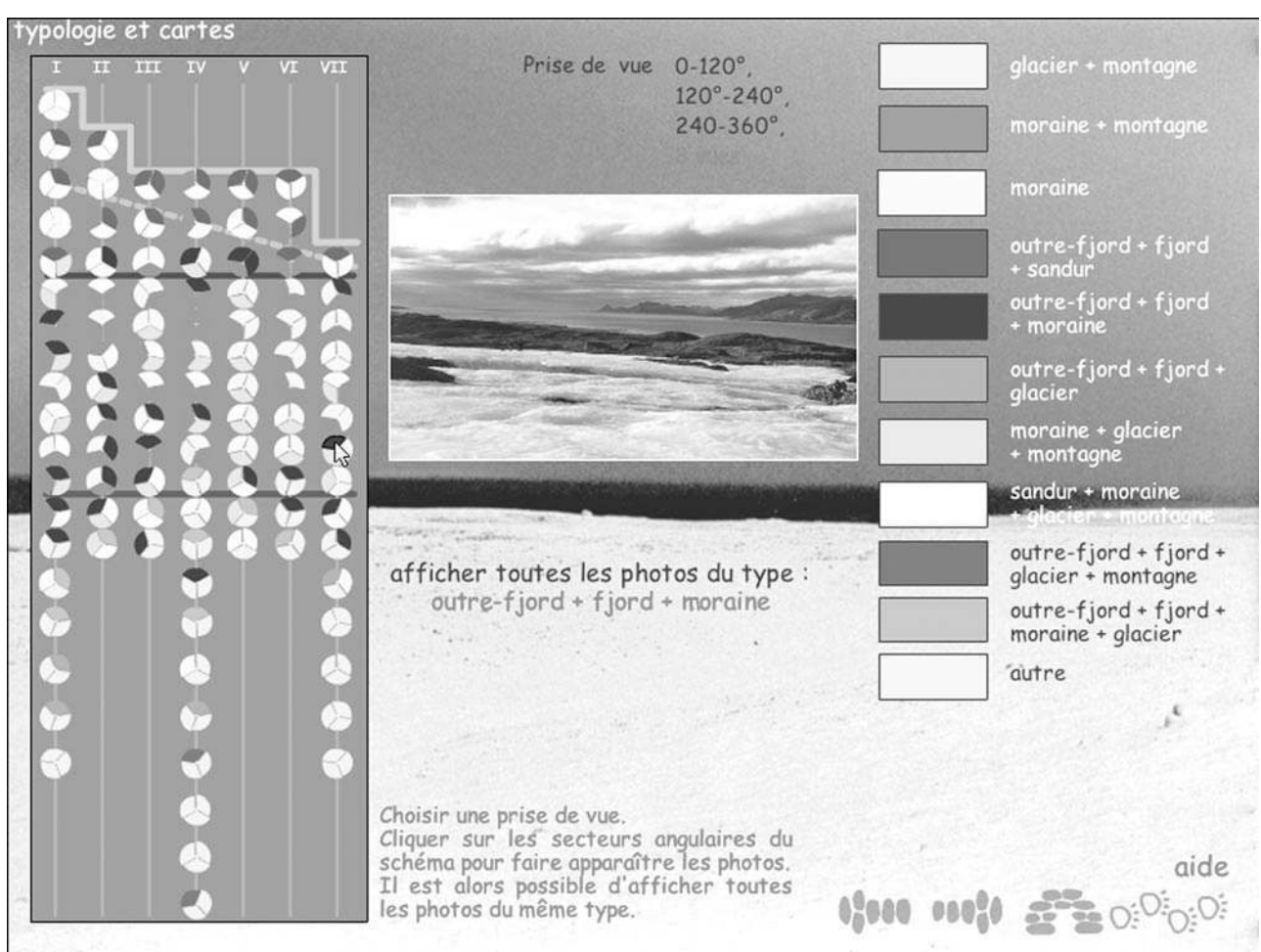

On peut aussi se demander de quel point de prise de vue est visible tel ou tel élément de base du paysage? le fjord? le glacier? la moraine? le sandur? l'outre-fjord? la montagne? (fig. 7). 
Figure 7 : De quel point et dans quelle direction peut-on voir le glacier? From which point and towards which direction can we see the glacier?
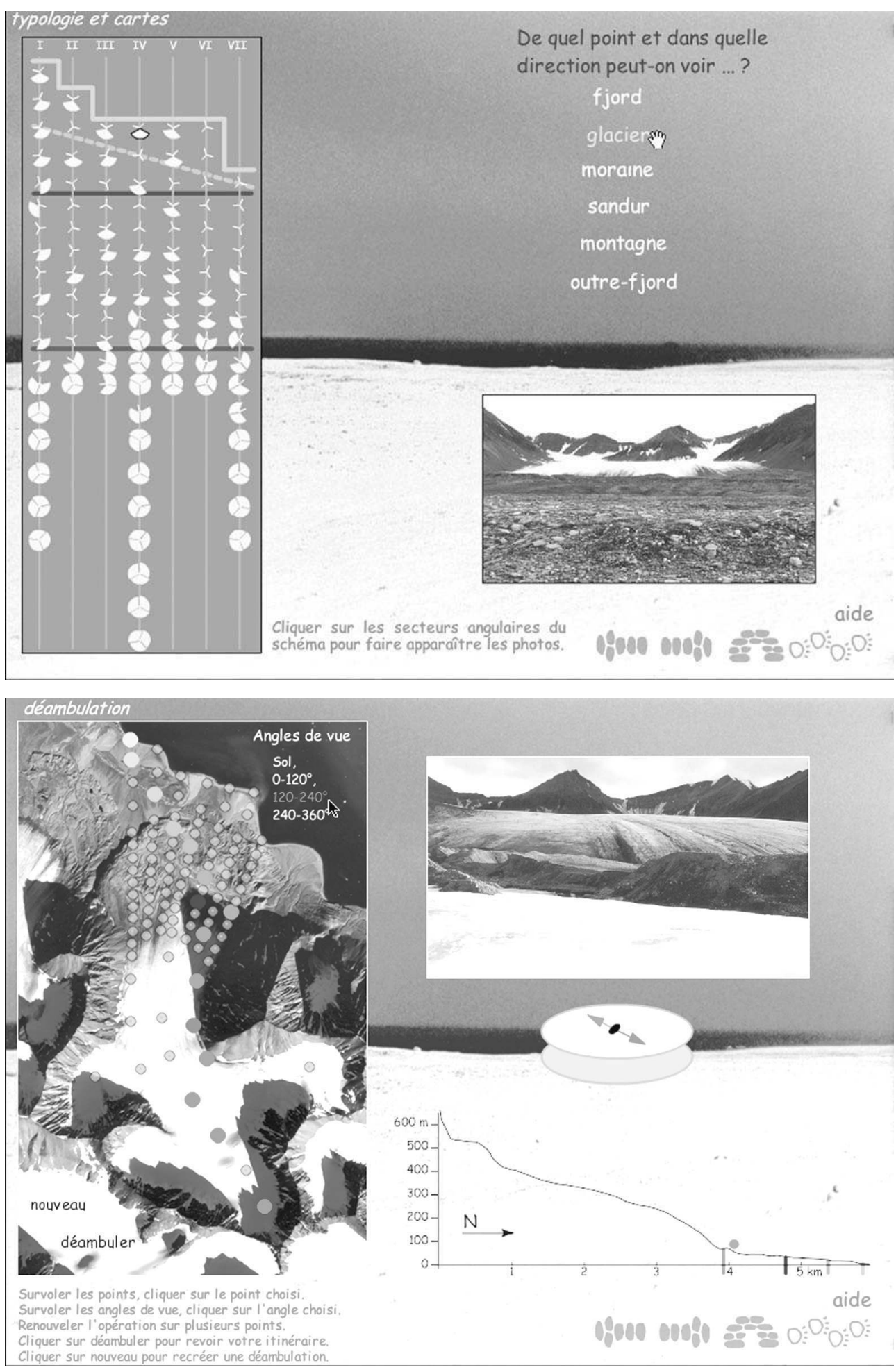

\section{Naviguer spatialement dans le paysage}

Le paysage est aussi déambulation, liberté $d u$ pas et du regard. Le thème "Cheminement» offre trois moyens de retrouver le continuum spatial du paysage. La 
navigation dans le paysage peut être réalisée de manière systématique le long des sept transects. On peut aussi visualiser trois itinéraires programmés permettant de retrouver les paysages lors d'une montée au glacier, d'une marche au bord du fjord ou d'un tour dans la moraine. Enfin, l'utilisateur peut simuler son propre cheminement, son propre regard, dans le module « Déambulation » et naviguer à l'infini dans le paysage du Lovén Est. La localisation du point sur la photographie aérienne et sur le profil en long permet un repérage instantané dans les trois dimensions de l'espace parcouru (fig. 8).

Figure 8 : Déambulation au cœur du bassin du Lovén Est Wandering through the East Lovén basin

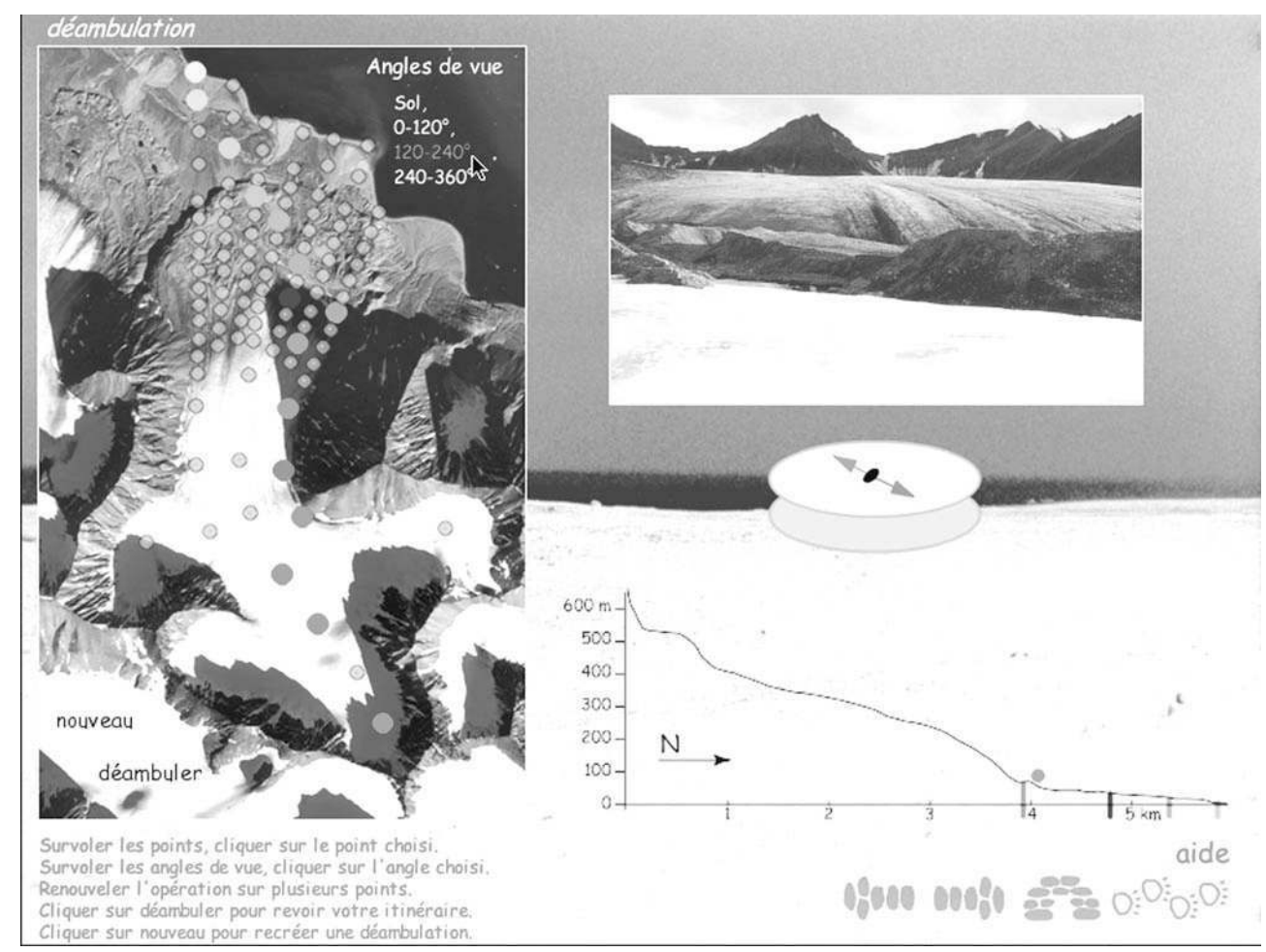

\section{Les temps du paysage}

Le thème « Au fil du temps » permet de naviguer dans les temps du paysage.

Un premier module offre une perception des changements du paysage à l'échelle de la saison : le long du transect central, 12 points ont fait l'objet de 3 passages d'observation en 50 jours. La banque d'images ainsi collectée permet des comparaisons globales sur l'ensemble du glacier (12 points), l'affichage par groupes de points ou point par point, pour les trois directions de prise de vue ou les trois passages successifs (fig. 9). 
Figure 9 : Variation temporelle le long de l'axe du bassin du Lovén Est Temporal variation of the landscape along the East Lovén basin axis

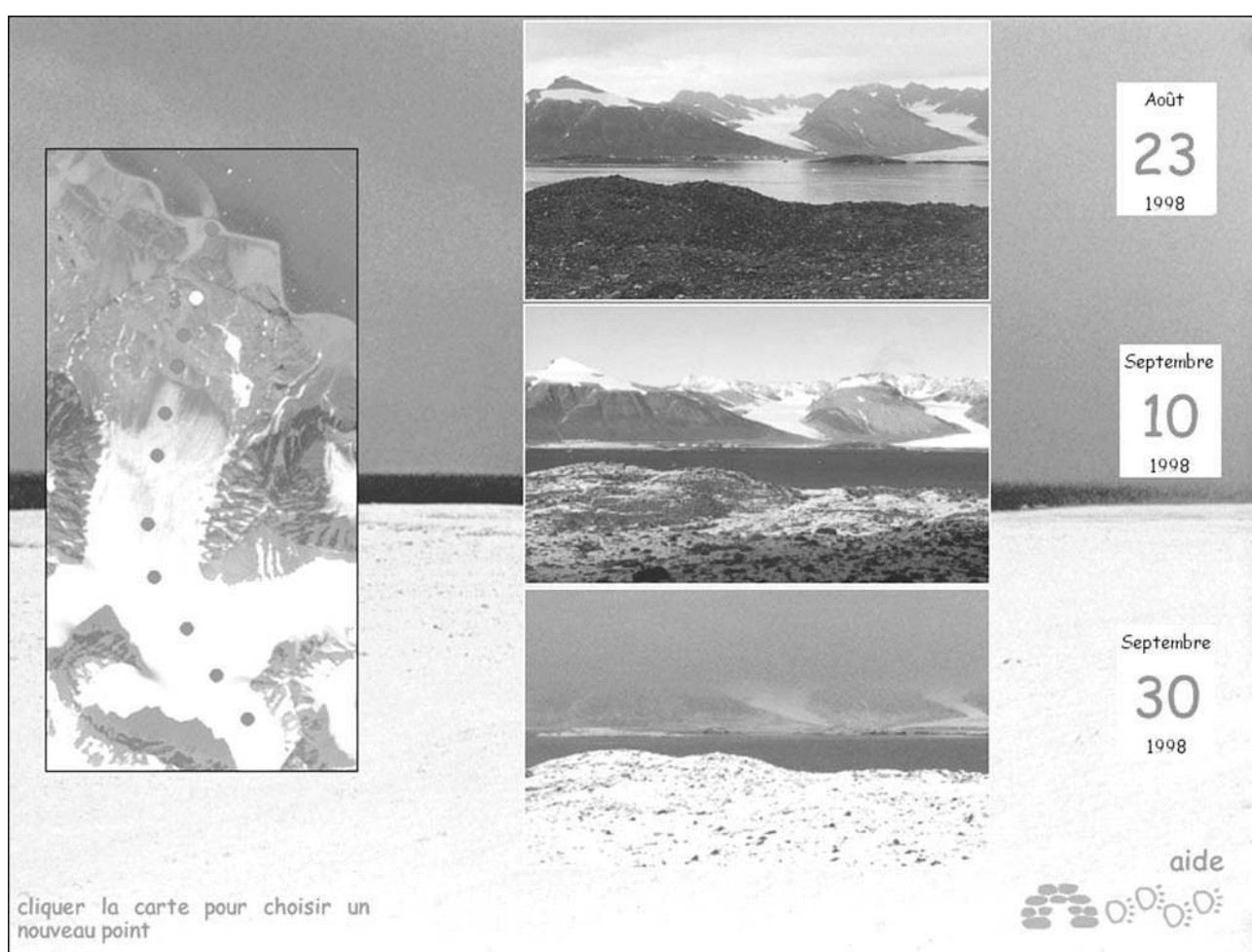

Le déroulement des saisons peut aussi être suivi au fil des jours ou des heures. Durant 50 jours, du point le plus proche de la base française, trois clichés ont été réalisés, chaque matin à $10 \mathrm{~h} 00$ : en direction du glacier du Roi, du Lovén Est, du cap Mitra. Pour accéder à la banque d'images temporelles, on peut choisir l'orientation, le pas de temps et le point de départ (fig. 10). 
Figure 10 : Variation journalière du paysage depuis la base française (pas de temps : 1 jour) Temporal variation of the landscape along the East Lovén basin axis (time step: 1 day)

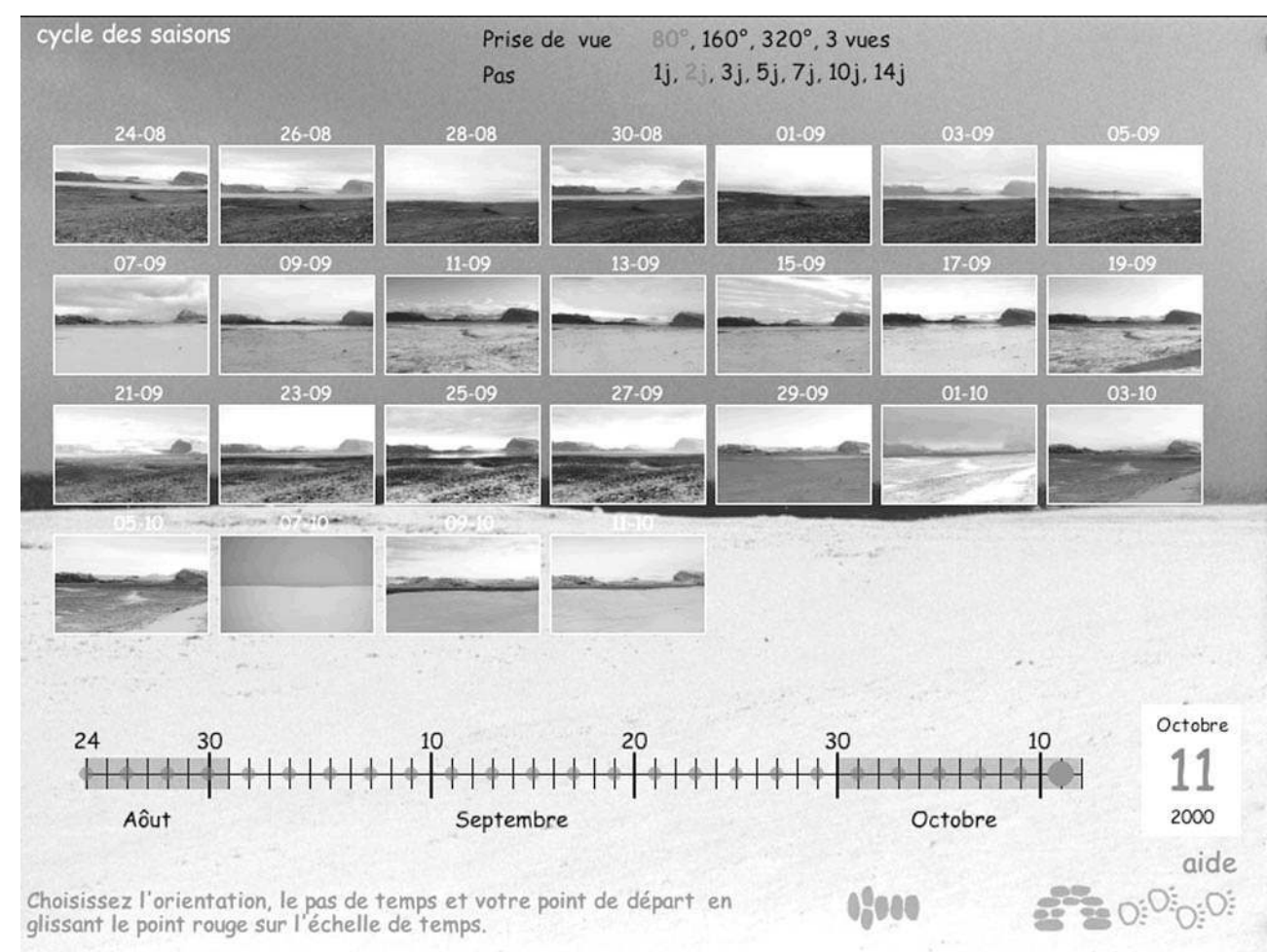

Pour deux journées (dernier soleil de minuit et première nuit sensible), la variation temporelle du paysage a été suivie de façon horaire : un écran de navigation semblable au précédent permet de mesurer l'importance de la lumière dans les paysages polaires (fig. 11). Ici, l'heure est l'unité du pas de temps. La comparaison est également possible entre plusieurs journées de suivi horaire.

Figure 11: Variation horaire du bassin du Lovén Est vu depuis la base française (pas de temps : 2 heures)

Hourly variation of the East Lovén basin seen from the French camp (time step : 2 hours) 


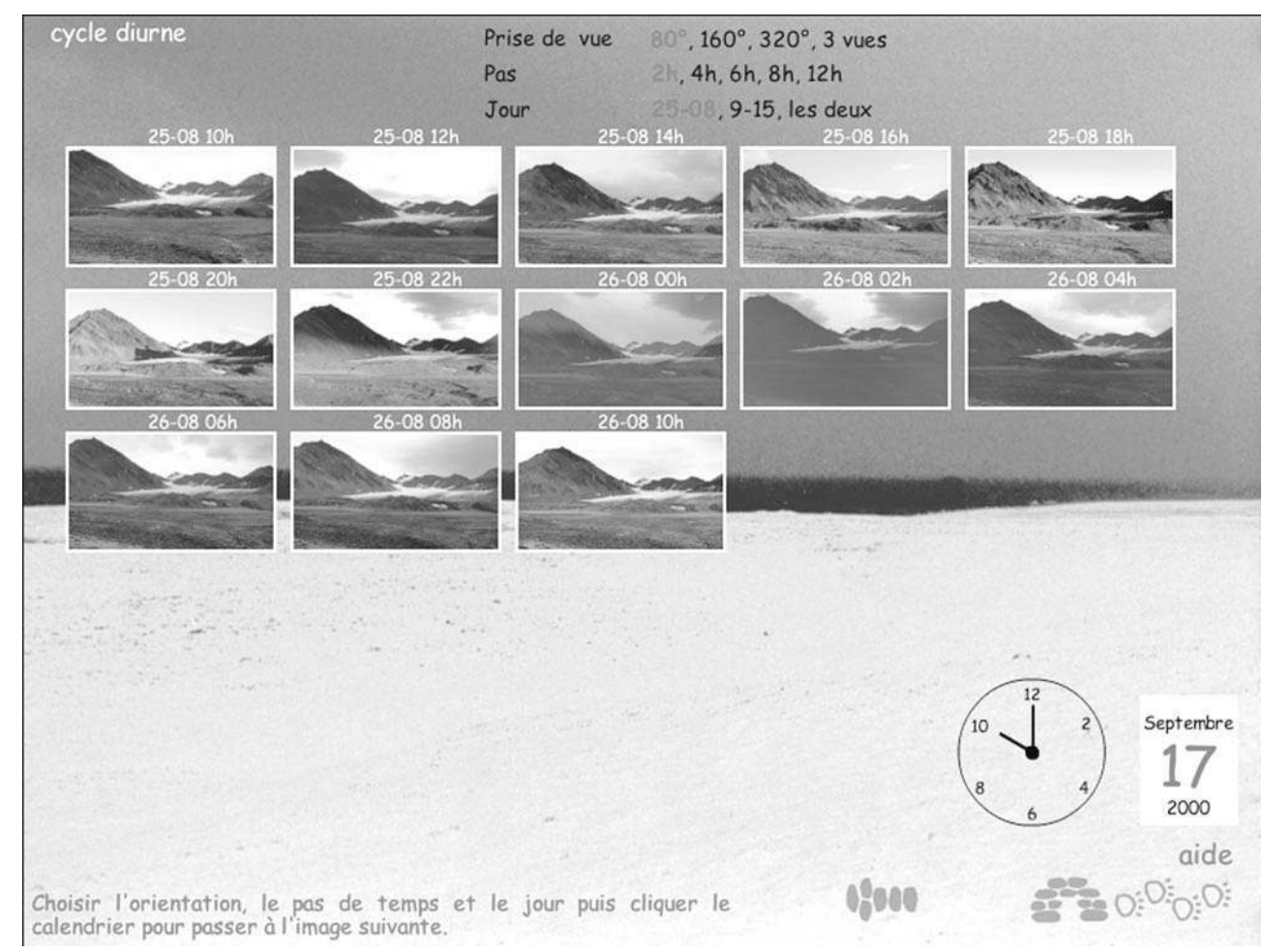

Le troisième niveau de navigation du cédérom permet diverses échappées : une approche des paysages moins en vue, tels ceux rencontrés dans les antres du glacier ( « Au cœur du glacier »), une évocation artistique à travers des aquarelles («Regard de peintre ») et des photographies ("Expo photo»). On peut également naviguer dans une banque d'images extraites de celles fournies par la webcam installée au sommet du Zeppelin qui offre, depuis quatre ans, un suivi temporel à l'échelle de la baie du Roi (« Un an en baie du Roi »).

\section{Conclusion}

Nous avons présenté ici un positionnement scientifique, il s'appuie sur un principe, celui que le paysage se voit depuis le sol, donc qu'il doit être étudié depuis le sol; sur une méthodologie, celle du continuum spatial, du carroyage, de la matrice d'information ; sur une filière technique, celle de l'échantillonnage photographique, de la banque d'images géoréférencées, de la navigation à travers des systèmes d'information conviviaux. En déroulant ce dispositif, nous avons voulu montrer qu'ainsi le paysage pouvait devenir un objet scientifique à part entière, ce qui n'est pas acquis, en particulier dans les milieux polaires où le paysage est souvent un point aveugle, évoqué dans les avants-propos ou réduit au rang d'illustration pour l'analyse d'autres phénomènes. Avant même l'exploitation de cette information paysagère, l'approche systématique modifie le regard que l'on porte sur le milieu en créant à la fois de la distance et de la proximité par rapport à lui. Le travail de terrain réalisé avec des chercheurs plus habitués à l'approche naturaliste nous l'a maintes fois montré. Cela dit, l'établissement d'une réalité-paysage conduit aussi à remonter vers l'amont du processus paysager. La banque d'images et les multiples possibilités d'accès et de navigation qu'elle autorise est aussi un remarquable instrument pour mobiliser le paysage-indice. La puissance informationnelle des scènes paysagères en perspective et la grande lisibilité des paysages arctiques font de ces images 
un moyen d'accès très efficace à la connaissance des processus glaciologiques, hydrologiques, climatologiques, etc. À condition toutefois que de véritables outils et procédures d'accès à l'information paysagères soient mis en œuvre.

Enfin, le paysage apparaît comme un efficace vecteur d'interaction entre chercheurs de différents champs scientifiques, ce qui est particulièrement bienvenu dans les zones polaires comme le Spitsberg où se côtoient souvent, sans forcément se rencontrer, des spécialistes de diverses disciplines. La nature fondamentalement systémique du paysage n'est pas étrangère à cela. Mais le paysage peut aussi être support de communication entre chercheurs et grand public, et à terme, plate-forme de médiation entre décideurs et ce même grand public.

Les perspectives entrevues pour ce travail sont nombreuses. Du côté de l'approche physionomique, des comparaisons avec d'autres espaces de l'Arctique permettront de mieux dégager les spécificités, de hiérarchiser les échelles, de clarifier la question des marges et des gradients. Du côté des dynamiques paysagères, les études multitemporelles sont à développer, à la fois pour la connaissance des rythmes et ruptures du paysage et pour la compréhension des processus en action dans cette partie du monde si réactive aux changements planétaires. Enfin, du côté de l'instrumentation, la mise au point d'une véritable télédétection paysagère à partir du sol, sur des terrains équipés d'appareils de prise de vue automatiques judicieusement placés nous paraît être riche d'avenir, particulièrement dans ces milieux extrêmes où l'observation directe sur le terrain n'est pas réalisable en continu mais où l'abandon de matériel coûteux en pleine nature demeure possible.

\section{BIBLIOGRAPHIE}

ANDRÉ (M.-F.), 1993. - Les versants du Spitsberg - Approche géographique des paysages polaires, Nancy, Presses Universitaires de Nancy, coll. « Géographie et Environnement », 361 p.

BORODKINE (R.), GRISELIN (M.), ORMAUX (S.), 2001. - « Towards a Landscape Information System », dans actes du colloque GeoComputation 2000, 5th International Conference on GeoComputation, Chatam Maritime (GB), University of Greenwich, 23-25 August 2000 (publication support interactif + site : [http://www.geocomputation.org/2000/GC012/Gc012.htm]).

Brossard (Th.), Joly (D.), ORMAux (S.), Wieber (J.-C.), 1998. - « Les paysages en question », Sciences et techniques de l'Environnement, 1, Besançon, Presses Universitaires Franc-comtoises, p. 7-14.

BRUnET (R.), Dollfus (O.), 1990. - Géographie universelle : Mondes nouveaux, Paris, Belin-Reclus, 480 p.

Griselin (M.), MARlin (Ch.), Moreau (L.), Dever (L.), 1995. - « Hydrologie et géochimie du glacier Lovén Est (Spitsberg) ", Actes du $3^{e}$ symposium Cavités glaciaires et cryokarst en régions polaires et de hautes montagnes, article bilingue français-anglais, p. 61-76. 
GRISELIN (M.), ORMAUX (S.), 2000. - « Les nouveaux médias pour une nouvelle culture du paysage », dans Multimédia et Construction des savoirs, actes du colloque international, Besançon 25-28 mai 1999, PUFC, p. 65-78.

-, 2001a. - « Analyse systématique du paysage visible à partir de photographies au sol : exemple du bassin Lovén Est, baie du Roi, Svalbard ", Actes des $4{ }^{e s}$ rencontres de Théo Quant, Besançon, 11-12 février 1999, p. 199-210.

-, 2001b. - «Le sens par la navigation : le multimédia pour expliquer le paysage », dans Actes du colloque Géopoint 2000, Avignon, 29-30 mai 2000, p. 137-148.

-, 2003. - « Le paysage et ses temporalités en baie du Roi, (Svalbard) : essai de problématisation et d'instrumentation », Bulletin de l'AGF : Séance de l'Association de Géographes Français « La recherche actuelle dans les milieux polaires et subpolaires ", Paris, 11 mai 2002, BAGF 2003-4, p. 386-393.

-, 2005. - Paysages vus du sol, principes d'analyse systématique : application en milieu arctique (Spitsberg), cédérom trilingue (français, anglais, espagnol). Prix « Enseignement de la recherche » du Festival du film de chercheur de Nancy, 2002.

Joly (D.), 2004. - « Ambiances climatiques instantanées : application à la microclimatologie du Spitsberg », Norois, $\mathrm{n}^{\circ}$ 191, p. 43-45.

ORMAUX (S.), 1987. - « Cartographie du paysage », Mappemonde, n 4, p. 28-33.

PÉGUY (Ch.-P.), 1969. - Ces montagnes qui flottent sur la mer, Paris, Arthaud, 318 p.

Pinchemel (Ph.) et Pinchemel (G.), 1997. - La face de la terre, Paris, Armand Colin, 524 p.

RAYNOLDS (M. K.), WALKER (D. A.), 2004. - « The circumpolar arctic vegetation map : analysis of distribution and NDVI vegetation types ", 8th CircumpolarSymposium on Remote Sensing of Arctic Environments, Chamonix, 6-12 juin 2004 : publication à paraître dans Remote Sensing of environment [http://thema.univ-fcomte.fr/circumpolar/list-presentations.html].

\section{RÉSUMÉS}

L'article suivant aborde les paysages polaires à travers la production récente d'un cédérom consacré à l'analyse de paysages depuis le sol en milieu arctique. Après avoir rappelé brièvement l'ambiguïté des relations entre géographie et paysage, on évoque l'évolution des représentations des paysages et des zones polaires chez nos contemporains, avant de montrer comment les nouveaux médias autorisent une approche renouvelée des paysages. Le cédérom, sur lequel on focalise progressivement le propos, sert de fil conducteur à cette réflexion sur le paysage polaire, considéré tout à la fois comme objet d'étude et comme espace expérimental.

This paper adresses the polar landscapes by the mean of a recently producted CD-Rom dedicated to arctic regions landscape analysis from the ground. After a brief reminder of the ambiguity of the relations between geography and landscape, we evoke the evolutions of the current representations of the landscapes and the polar regions, then we show how the new media permit a renewed approach of the landscapes. The CD-Rom presented here is the support to this reflection about polar landscape, considered as object of study and experimental area. 
INDEX

Index géographique : Arctique, Spitsberg

Mots-clés : analyse systématique, banque d'images, multimédia, paysage

Keywords : images bank, landscape, multimedia, systematic analysis

\section{AUTEURS}

\section{MADELEINE GRISELIN}

Université de Franche-Comté, THÉMA, UMR 6049 - CNRS, madeleine.griselin@univ-fcomte.fr

\section{SERGE ORMAUX}

Université de Franche-Comté, THÉMA, UMR 6049 - CNRS, serge.ormaux@univ-fcomte.fr 